\title{
Application of nonlinear fatigue damage models in power electronic module wirebond structure under various amplitude loadings
}

\author{
Pushparajah Rajaguru $\cdot$ Hua Lu $\cdot$ Chris Bailey
}

Received: 19 September 2013/Accepted: 20 January 2014/Published online: 28 March 2014

(C) Shanghai University and Springer-Verlag Berlin Heidelberg 2014

\begin{abstract}
This paper presents mean fatigue lifetime prediction of a wire-bond structure model in power electronic module using a failure physics approach that integrates high fidelity modelling and reduced order modelling. Loading current with variable amplitudes is applied to a finite element model of simplified wirebond structures. The resulting accumulated fatigue damage due to random loads is predicted by using reduced order modelling based on failure physics, a cycle counting algorithm, and various nonlinear fatigue damage models widely used in the literature. The reduced order modelling approach based on failure physics uses prediction data for the electro-thermo-mechanical behaviour of the wire-bond design of a power module obtained through non-linear transient finite element simulations, in particular for the fatigue life-time of the aluminium wire attached to the silicon chip of the wire in the module. The reduced order models that capture the black box function of the accumulated plastic strain are used in predicting the mean fatigue life time of the wire bond structure under random loads. One of the widely used cycle counting algorithms, rainflow counting algorithm, is used to count cycles of the temperature profile at the specific point of the wire bond structure in a power electronic module. The cycle data from the rainflow algorithm mean life time of the wire bond structure are predicted with various cumulative fatigue models. Non-linear cumulative fatigue models such as damage curve approach (DCA), double linear damage rule (DLDR), and double damage curve approach (DDCA), and linear cumulative fatigue damage model such as Palmgren-Miner rule are used to predict the mean fatigue life of the wire bond structure, and the results are compared.
\end{abstract}

P. Rajaguru $(\varangle) \cdot$ H. Lu · C. Bailey

Computational Mechanics and Reliability Group,

University of Greenwich, London, UK

e-mail: p.r.rajaguru@gre.ac.uk
Keywords Reduced order models - Power module . Kriging $\cdot$ Radial basis $\cdot$ Damage rule

\section{Introduction}

Power electronic modules (PEMs) are self contained power electronics components, which are widely used in various industries such as aerospace, automobile, etc. PEMs have highly inhomogeneous structure, which consist of several insulator or ceramic layers, conductor and semiconductor, some metal wires, and other materials. These materials are assembled in the packaging process to form the power electronic circuit, as shown in Fig. 1. PEMs usually dissipate large amounts of heat, and operate in harsh environment such as engine compartments of automobiles. Nowadays PEMs require far greater reliability with respect to electric performance, insulation efficiency, thermal performance, and robustness.

The failure mode in PEMs associated with the wire bond lift-off is one of the major issues. Aluminium wires with diameter up to $500 \mu \mathrm{m}$ connect the silicon chip with the output pins of power electronic packages. It is compulsory to use many wires to handle the electric current of up to 75 A per chip. Each wire is bonded on thin aluminium pads of some micron thickness. Wire bond connections are susceptible to fatigue as a result of thermo-mechanical damage mode during the power operations. Wire bond liftoff is regarded as the dominant failure mode caused by the power cycles, which leads to a non-homogeneous current distribution on the power module chip and higher local and average temperatures. These increase the lifting process of further wires in a power module [1].

Finite element modelling (FEM) has been extensively applied to study the wirebond failure in PEMs [3-5]. 


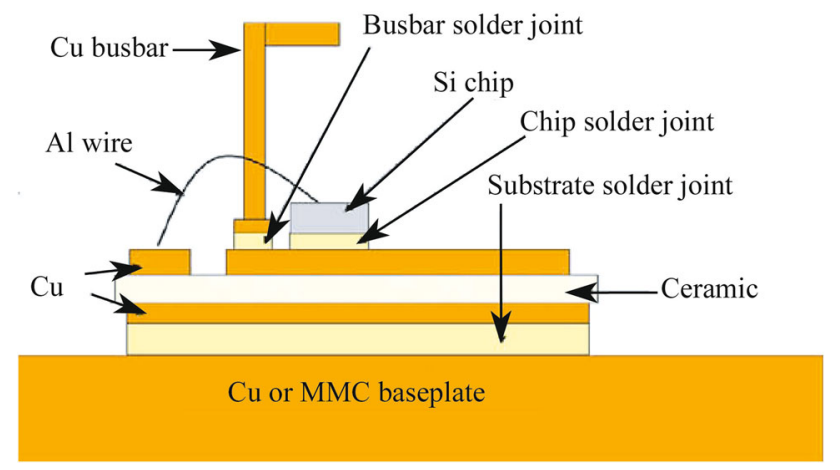

Fig. 1 Cross sectional view of power module, courtesy of Ref. [2]

A wirebond heel region failure mechanism and its finite element analysis (FEA) mechanical simulation are reported in Ref. [6]. Hager [7] conducted a similar detailed study for lifetime estimations of thick aluminium bond wires in IGBT power modules under environment conditions by utilizing FEA model to measure the elasto-plastic behaviour of the aluminium wire. Another FEA study to analyze the thermo-mechanical behaviour of the wire bonds in electronic packages is reported by Van Driel et al. [8]. The mechanical FEA for flexural failure of wires and the associated lifetime prediction models are reported by Meyyappan et al. [9] for various wire curvatures at the heel of the wire.

This paper focuses on analyzing the accumulated fatigue life of wirebond structure under variable amplitude electric loading profiles using electro-thermo-mechanical analysis in FEM setup. Some literatures $[10,11]$ discussing wirebond model under the random loading condition have the same principle as applying a cycle counting algorithm and linear damage model. In this paper a comparison study using nonlinear damage models as well as linear damage model is utilized by using cycle counting algorithm and physics of failure $[12,13]$ for wirebond structure failure mode in electro-thermo-mechanical finite element simulation. The comparison study utilizes four widely used nonlinear fatigue damage models.

\section{Cumulative fatigue damage models}

Fatigue is one of the primary failures, which increases with applied loading cycles in both constant loading and variable loadings. Damage accumulation by variable amplitude loadings (often referred as spectral loading) differs from that by constant amplitude loading. Accumulation of fatigue damage for applied load cycles of variable amplitude loadings often processes in a cumulative manner, which eventually causes the fracture. For more than eight decades, various researchers have tried to find a superior method to predict the damage accumulation behaviours. A full comprehensive review on most of the fatigue damage accumulation methods can be found in Ref. [14]. In this paper, only four damage accumulation rules are discussed and utilized to predict the fatigue life of the aluminium wire bond model under random loadings due to widespread usage of these models. There are many parameters in the fatigue damage models utilized in this study based on the available data in the literatures.

\subsection{Linear damage rule (LDR)}

The most popular method of accumulating the damages for random load profiles is LDR commonly referred as Palmgren-Miner rule, which was proposed by Palmgren in 1924 and later developed by Miner in 1945 [15]. In terms of mathematics, the LDR can be defined as follows:

$D=\sum_{j=1}^{i} \frac{n_{j}}{N_{j}^{\mathrm{f}}}$

where $D$ denotes the damage, and $n_{j}$ and $N_{j}^{\mathrm{f}}$ are the applied cycle numbers of operations and total cycles to failure under $j$ th constant amplitude load levels, respectively. Fatigue failure is predicted to occur if

$\sum_{j=1}^{i} \frac{n_{j}}{N_{j}^{\mathrm{f}}} \leq c$

where $c$ is a parameter modelling accuracy of the applied damage accumulation model within the range $0.7-2.2$ in practice, which is obtained by experiments. The high variability in the parameter $c(0.7-2.2)$ indicates the high model uncertainty. When there is no information available, the constant $c$ is set to one. Because of the simplicity, it is still widely used. However several significant influences are ignored, and there will be failure prediction errors because of the uncertainty. The primary drawback of LDR is its inability to account for the order of loading. Subsequently failure prediction by this method does not take into account the effects of loads interactions experimentally observed.

\subsection{Damage curve approach (DCA)}

The DCA approach has been proposed by Manson and Halford [16]. They proposed the damage approach for computing the fatigue damage in a cycle by cycle manner. It was fundamentally assumed that significant number of cycles had to be applied before enough damage could accumulate to cause a reduction in life. The amount of accumulated damage $D$ for a material with initial crack 
length $a_{0}$ and crack length at fracture of $a_{\mathrm{f}}$ at a given load level that results in crack length of $a$ can be presented as

$D=\frac{a}{a_{\mathrm{f}}}=\frac{1}{a_{\mathrm{f}}}\left(a_{0}+\left(a_{\mathrm{f}}+a_{0}\right) \frac{n}{N_{\mathrm{f}}}\right)^{B}$,

$B=\frac{2}{3} N_{\mathrm{f}}^{0.4}$,

where $D$ accumulates to one, and the damage material reaches the end of its usual life. When initial crack length $a_{0}$ is assumed zero and $a_{\mathrm{f}}$ is assumed to be one, the final form for the fatigue damage $D$ is derived as

$D_{i}=\left(\frac{n_{i}}{N_{\mathrm{f}}^{i}}\right)^{\left(\frac{N_{\mathrm{f}}^{i}}{N_{\mathrm{Ref}}}\right)^{0.4}}$,

where $N_{\text {Ref }}$ is the reference fatigue life typically taken as one. Total accumulated fatigue damage by DCA is as follows :

$D=\sum D_{i}=\sum\left(\left(\frac{n_{i}}{N_{\mathrm{f}}^{i}}\right)^{\left(\frac{N_{\mathrm{f}}^{i}}{N_{\mathrm{ref}}}\right)^{0.4}}\right)$.

The primary advantage of employing the DCA model is its ability to create identical damage curves for different reference fatigue lives [16].

\subsection{Double LDR (DLDR)}

DLDR proposed by Manson in 1966 is based on crack initiation and crack progression state and applies the LDR to predict the total lifetime [17]. The double linear damage curve for various loading amplitudes can be interpolated in terms of two distinct states based on the minimum and maximum fatigue lives of the block. The total fatigue life $N^{\mathrm{f}}$ is the summation of the state 1 fatigue life $\left(N^{1}\right)$ and state two fatigue life $\left(N^{2}\right)$. Therefore failure is predicted, once the two states satisfy the LDR in sequence.

$\sum D_{j}^{1}=\sum_{j=1}^{i} \frac{n_{j}}{N_{j}^{1}}=1$

$\sum D_{j}^{2}=\sum_{j=1}^{i} \frac{n_{j}}{N_{j}^{2}}=1$

$N^{\mathrm{f}}=N^{1}+N^{2}$.

The interpolation formula to generate bilinear damage model based on Manson and Halford [17] is as follows:

$N^{1}=N^{\mathrm{f}} \mathrm{e}^{A\left(N^{\mathrm{f}}\right)^{B}}$.

where $A$ and $B$ are
$A=\frac{\ln \left(0.35\left(\frac{N_{\text {Low }}}{N_{\text {High }}}\right)^{0.25}\right)}{N_{\text {Low }}^{B}}$,

$B=\frac{1}{\ln \left(\frac{N_{\text {Low }}}{N_{\text {High }}}\right)} \ln \left(\frac{\ln \left(0.35\left(\frac{N_{\text {Low }}}{N_{\text {High }}}\right)^{0.25}\right)}{\ln \left(1-0.65\left(\frac{N_{\text {Low }}}{N_{\text {High }}}\right)^{0.25}\right)}\right)$.

$N^{2}=N^{\mathrm{f}}-N^{1}=N^{\mathrm{f}}\left(1-\mathrm{e}^{A\left(N^{\mathrm{f}}\right)^{B}}\right)$,

where $N_{\text {Low }}$ and $N_{\text {High }}$ are the lowest and highest fatigue lives of the block. The material constant values 0.35 and 0.25 are taken in this study [18].

\subsection{Double damage curve approach (DDCA)}

This method slightly varies from the DCA by having a linear term to the original DCA equation with some extra mathematical terms. DDCA approximates the DLDR in the lower life region and DCA in the higher life region. The DDCA is defined as follows [19]:

$$
\begin{aligned}
D_{i} & =\frac{n_{i}}{N_{i}}\left(q_{i, 1}^{C}+\left(1-q_{i, 1}^{C}\right)\left(\frac{n_{i}}{N_{i}}\right)^{C\left(q_{i, 2}-1\right)}\right)^{1 / C}, \\
q_{i, 1} & =\frac{0.35\left(\frac{N_{\mathrm{Ref}}}{N_{i}}\right)^{A}}{1-0.65\left(\frac{N_{\mathrm{Ref}}}{N_{i}}\right)^{A}}, \\
q_{i, 2} & =\left(\frac{N_{i}}{N_{\mathrm{Ref}}}\right)^{B},
\end{aligned}
$$

where $D_{i}$ is the damage accumulated at the $i$ th load profile, $n_{i}$ the number of applied cycles at the $i$ th load profile, $N_{i}$ the number of fatigue life cycles to fail at the $i$ th amplitude load. $C$ is set to 5 , and $A$ and $B$ are material constants which are typically taken as 0.25 and 0.4 respectively for many alloys [19].

\section{FEA of wire bond structure}

The FEA computer modelling method investigating the effect of varying thermal loads due to applied random loading currents on the aluminium wire is simulated. The residual stress in the wirebond is obtained. To simulate the electro-thermo-mechanical loading condition on a wire bond, the dimensional data of a wire bond [20] are used in ANSYS [21] FEA software to generate the finite element model, as shown in Fig. 2. This model contains a slice of the device along the wire and uses the periodic boundary conditions to represent the effects of the array of wires. Mirror plane symmetry of the structure is taken so that only 

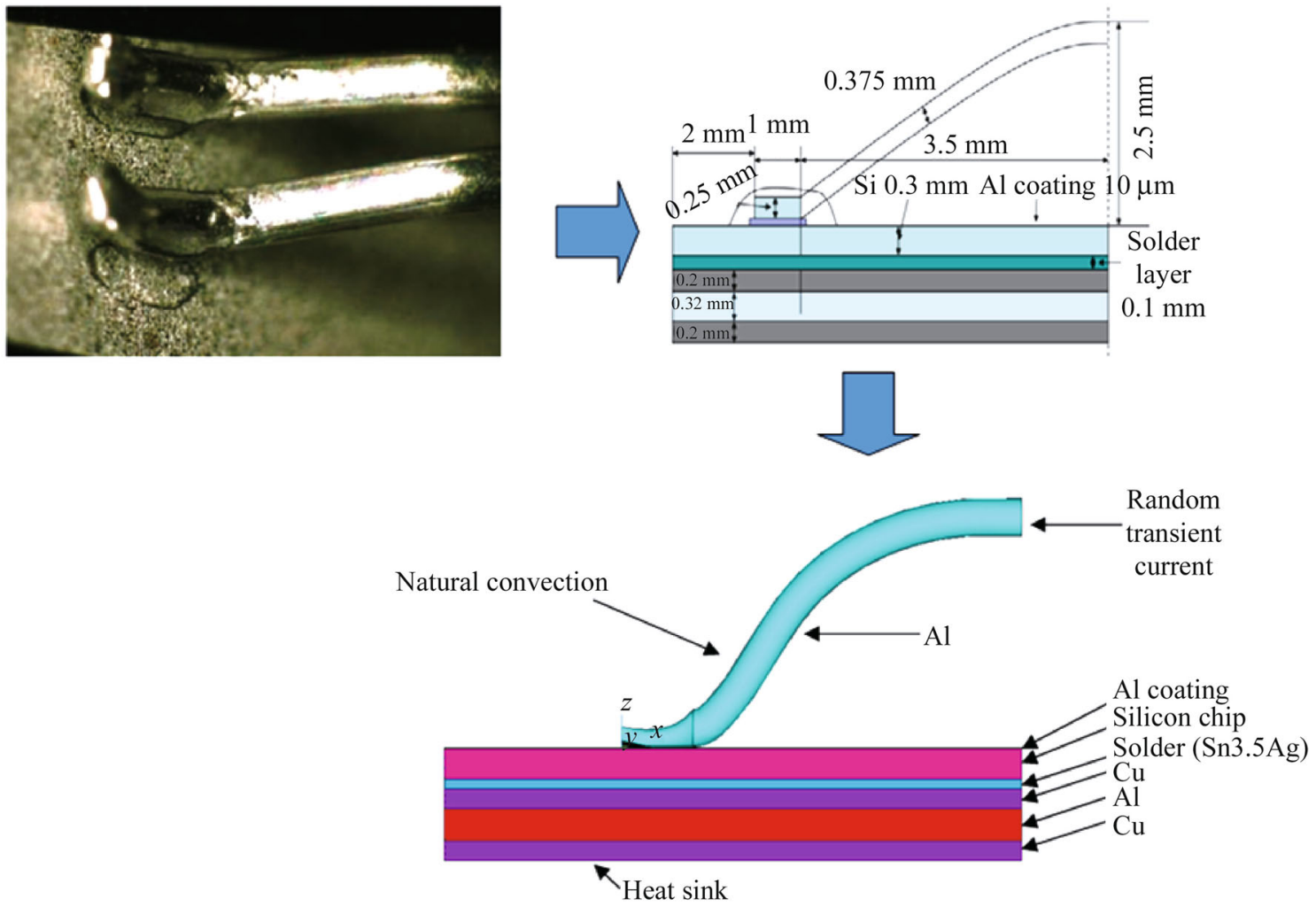

Fig. 2 Dimensions of the wirebond model [19] and the resulted FEA computer simulation

half of the wire and surrounding structure are included in the model to reduce the computational complexity. This model does not take into account the location of the wire in the PEM device and its influence.

The studied model consists of silicon chip, ceramic substrate and aluminium wire. Chips are soldered on ceramic substrates which consist of aluminium nitride with thin layer of copper $(0.2 \mathrm{~mm})$ bonded on both sides by high temperature oxidation process. The ceramic material ensures the electrical insulation and lower copper layer serves as heat sink during power operation. Lower copper layer is mounted on a cooling unit or heat sink. An aluminium metallization is used between aluminium wire and silicon chip. The design of high power electronic module comprises of parallel connection of many chips. The chips are soldered to the ceramic substrate aluminium wires that connect the chips with output pins of the power electronic packages. Each wire is bonded on thin aluminium of some micron thickness on the silicon chip. Thickness of all materials used in this model is shown in Fig. 2.

The elastic and thermal material properties of all the materials used in this model are listed in Table 1. The plastic material properties of aluminium are listed in Table 2. Figure 2 shows the geometry and material behaviours of the different layers. The parts in the model associated with critical regions of interest have fine meshes in order to ensure accurate FEA results. In this study, the indirect coupling method in ANSYS is utilized. In indirect coupling method, the first electro-thermal FEA is simulated using SOLID 69 element, then the results from the electrothermal analysis are uploaded to structural FEA using the element switching procedure in ANSYS. Numerical modelling approach such as FEM presented in this paper to capture the involved complex physics refers to the high fidelity modelling.

Aluminium coating and aluminium wire are exposed to natural air open surface, which creates a natural convection for the model to dissipate heat generated from the model. Additionally the model has a heat sink at the base copper plate. We select the average convection coefficient of heat sink as 210 based on a previous study [22]. Convection coefficients for the aluminium wire and aluminium coating plate are defined as

$h_{\text {Wire }}=0.52\left(\frac{g \beta \Delta T L_{\mathrm{cl}}^{3} P_{\mathrm{r}}}{v^{2}}\right)^{1 / 4} \frac{k}{L_{\mathrm{cl}}}$,

$h_{\text {Al plate }}=0.15\left(\frac{g \beta \Delta T L_{\mathrm{p}}^{3} P_{\mathrm{r}}}{v^{2}}\right)^{1 / 3} \frac{k}{L_{\mathrm{p}}}$,

where $\beta$ the expansion coefficient, $P_{\mathrm{r}}$ the Prandtl's number, $k$ the thermal conductivity and $v$ the kinematic viscosity, 
Table 1 Elastic and thermal material properties used in the FEA

\begin{tabular}{llllll}
\hline & $\begin{array}{l}\text { Young's } \\
\text { modulus/GPa }\end{array}$ & $\begin{array}{l}\text { Poisson's } \\
\text { ratio }\end{array}$ & $\begin{array}{l}\text { Coefficient of thermal } \\
\text { expansion/ }\left(\mathrm{ppm} \cdot{ }^{\circ} \mathrm{C}^{-1}\right)\end{array}$ & $\begin{array}{l}\text { Thermal conductivity/ } \\
\left(\mathrm{W} \cdot \mathrm{m}^{-1} \cdot \mathrm{K}^{-1}\right)\end{array}$ & $\begin{array}{l}\text { Electrical resistivity/ } \\
(\Omega \cdot \mathrm{m})\end{array}$ \\
\hline $\mathrm{Al}$ & 70 & 0.33 & 24.5 & 235 & $2.65 \times 10^{-8}$ \\
$\mathrm{Si}$ & 113 & 0.29 & 3 & 150 & 0.0001 \\
$\mathrm{Cu}$ & 103.42 & 0.3 & 17 & 400 & $1.7 \times 10^{-8}$ \\
$\mathrm{Solder}\left(\mathrm{Sn}_{3} \cdot 5 \mathrm{Ag}\right)$ & $54.05-0.193 \mathrm{~T}$ & 0.4 & $21.85+0.02039 \mathrm{~T}$ & 54 & $1.3 \times 10^{-7}$ \\
$\mathrm{Al} \mathrm{nitride}$ & 310 & 0.24 & 5.6 & 160 & $1 \times 10^{14}$ \\
\hline
\end{tabular}

Table 2 Plastic material property of $\mathrm{Al}$

\begin{tabular}{lll}
\hline & Yield stress $\sigma / \mathrm{MPa}$ & Tangent modulus $E_{\mathrm{t}} / \mathrm{MPa}$ \\
\hline $\mathrm{Al}$ & 29 & 35.5 \\
\hline
\end{tabular}

$h_{\text {Wire }}$ irregular solid heat transfer coefficient, $L_{\mathrm{cl}}$ characteristic length of the wire, $h_{\mathrm{Al} \text { plate }}$ horizontal plate upper surface heat transfer coefficient and $L_{\mathrm{p}}$ ratio between the area of the plate exposed to air and the perimeter of the plate [23]. All these parameters are the characteristic values in air.

Mechanical boundary condition imposed on the model in the finite element simulation is the rectangular prism of the model flat surfaces with imposed displacement constraint. All sides of rectangular prism except the side which is connected to the wire of the model structure have the displacement constraint. The sideways of the wirebond model have zero displacement. Electrical transients loading current which is a random load is fed into the aluminium wire, and one side of the rectangular prism has zero voltage. Figure 3 shows the electric load current which is a ramped random load and the resulted approximate temperature profiles at the junction of aluminium wire and aluminium plate interface by FEA simulation. Additionally tensile and shear stresses due to bond wire fluctuations during the operation are not considered in this paper.

Figure 4 is the FEA output for plastic strain of the interface between the wire and the plate. The evaluation of predictive fatigue life time can be categorized based on stress, plastic strain, plastic and creep strain, energy, and damage accumulation during a test $[24,25]$. For aluminium wire bond, we use the inelastic strain based life prediction model which considers plastic strain caused by coefficient of thermal expansion mismatches between various materials in the model structure. The Coffin-Manson fatigue life model which is one of the widely used models is used as follows:

$N_{i}=C\left(\Delta \varepsilon_{\text {In }}\right)^{k}$,

where $\Delta \varepsilon_{\text {In }}$ is the accumulated equivalent inelastic strain during a stabilized cycle, which is used for aluminium wire bond fatigue life prediction. For the calculation of cycles number before failure, we use part of the volume of $17.5 \mu \mathrm{m}$ thickness of aluminium wire, which is an interface to the aluminium coating plate and aluminium wire, as shown in Fig. 4. By visually inspecting the global model for plastic strain, we decide to slice the wire at this thickness from the intersection. To calculate the accumulated inelastic strain, we use the widely reported method volume weighted average (VWA) shown as

$\Delta \varepsilon_{\text {In }}=\frac{\sum_{j} \Delta \varepsilon_{\text {In }}^{j} V_{j}}{V_{\text {Total }}}$,

where $V_{\text {Total }}$ the summation of volumes of all the elements, $V_{j}$ the volume of the $j$ th element, and $\Delta \varepsilon_{\text {In }}^{j}$ the associated accumulated inelastic strain. The various values are proposed for $C$ and $k$ in Eq. (17) based on the different types of aluminium. A study by Meyyappan et al. for aluminium alloy is found to predict the values of $C$ and $k$ as 1 and -1.4 [9]. These values are applicable in the case when elastic strain is small compared to the plastic strain range. It is assumed that the total strains (elastic and plastic) have been approximated to be equal to the plastic strain. This plastic strain is then used in the Coffin-Manson based relation (Eq. 17) to predict the cycles to failure. The input from FEA simulation in ANSYS during the power cycling test includes the accumulated plastic strain (ANSYS output parameters NL, EPEQ). A volume averaging technique described in Eq. (18) is then employed to calculate the accumulated plastic strain for $17.5 \mu \mathrm{m}$ thickness aluminium layer. The standard output from ANSYS (NL, EPEQ) provides the accumulated plastic strain, which can be averaged by post processing steps using ETABLES in ANSYS as proposed by Ref. [25].

\subsection{Rainflow cycle counting method}

Various cycle counting methods exist in the literature. All the counting methods count a cycle with the range from the highest peak to the lowest valley. Rainflow cycle counting is the most popular algorithm, which was first proposed by Matsuishi and Endo [26]. Therefore the method is called 

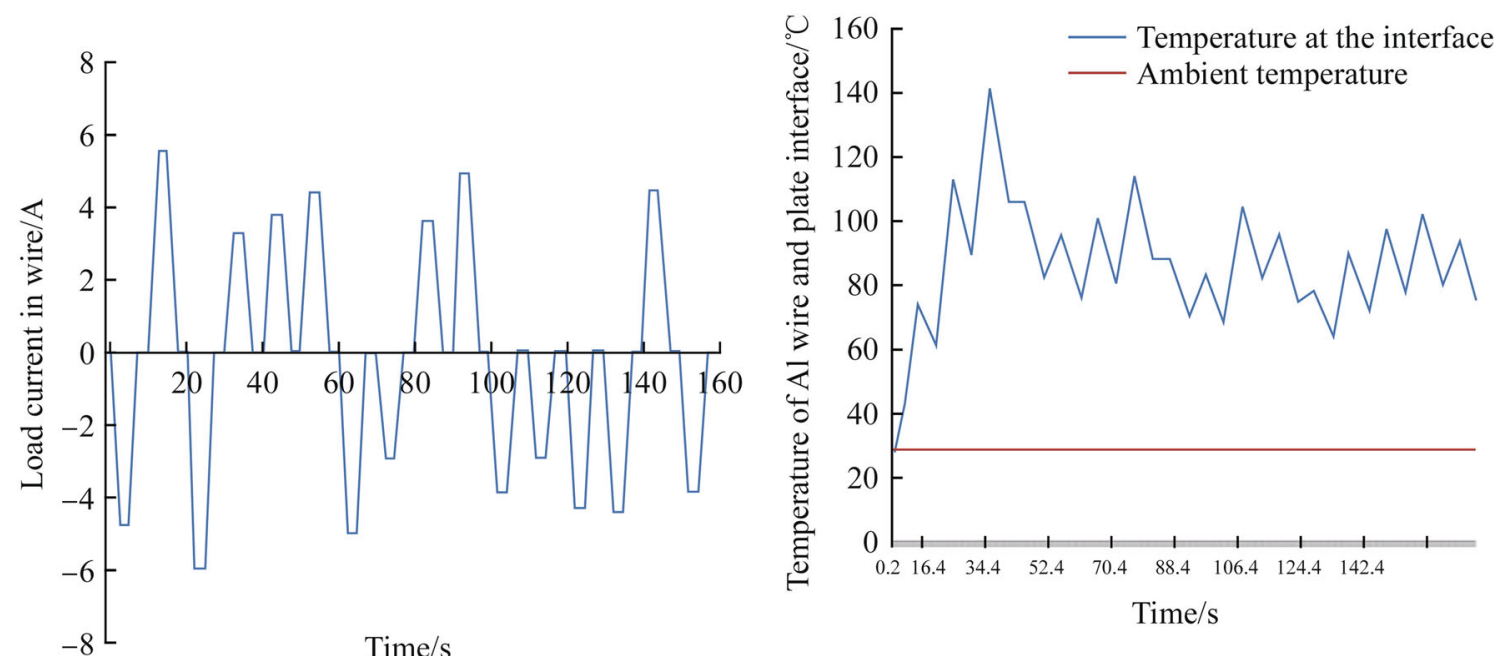

Fig. 3 Random load current profile applied to the aluminium wire and the resulting approximate temperature profile of the aluminium wire and aluminium plate interface

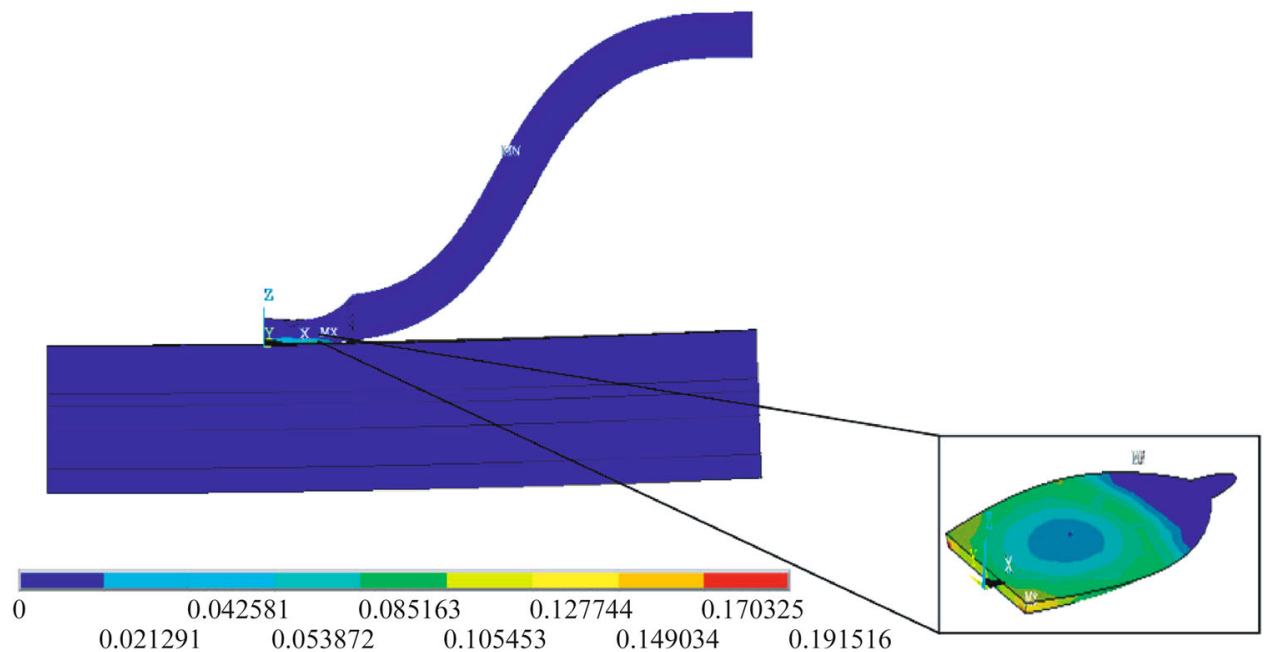

Fig. 4 Plastic strain of sliced part of the aluminium wire with $17.5 \mu \mathrm{m}$ thickness in the Al plate and $\mathrm{Al}$ wire interface

rainflow counting, as shown in Fig. 5. Various improvements of original version of rainflow cycle counting method are mentioned in literature, such as the simplified rainflow cycle counting method [27]. In this paper we use the original version as in Ref. [28]. Table 3 shows cycles counted from rainflow counting algorithm for the temperature profile at the $\mathrm{Al}$ plate and $\mathrm{Al}$ wire interface.

\section{Reduced order modelling (ROM) through data interpolation approach}

The ROM involves the formulation of models that can evaluate and analyze a system or process behaviour relatively faster without using significant computational resources and time. A ROM is typically an approximation of the true behaviour of the blackbox function that represents the system. The ROM strategy presented here uses data obtained through design of experiment (DoE) techniques [29]. The DoE is a specific way of obtaining the design points set in the design space. The models are developed as functions of the selected design variables for investigation. In this work, two interpolation-based models, Kriging and Radial basis functions using DoE are discussed. The models are demonstrated for the wire bond structure in the power electronic module. The discrete model function values corresponding to the system that depend on the design variables are obtained by DoE based sampling.

\subsection{DOEs}

The first step in the ROM approach is to derive performance characteristic data through DoE by evaluation of a 


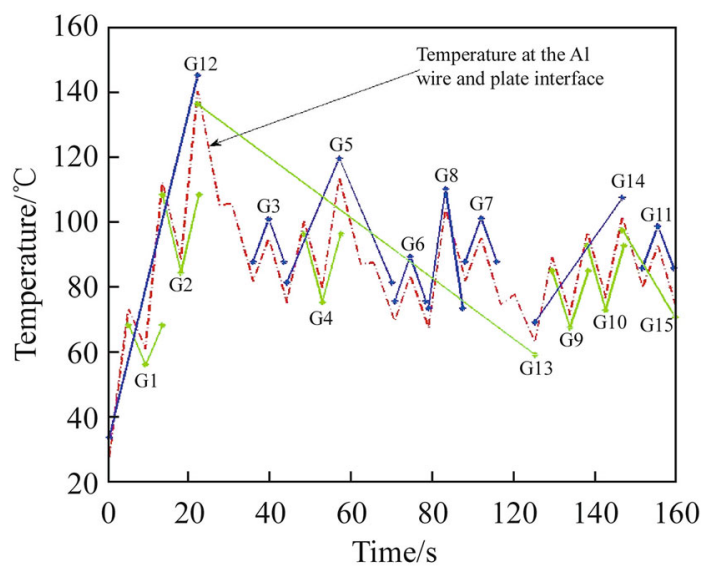

Fig. 5 Temperature profile with the associated cycles from rainflow algorithm

limited number of design scenarios. DoE methods are applied in the two-dimensional design space defined by the respective limits for the amplitude of temperature load profile $\left(10-57{ }^{\circ} \mathrm{C}\right)$ and mean value of temperature load applied to the wire $\left(67-102{ }^{\circ} \mathrm{C}\right)$ from Table 3 . The mean temperature value varies from $67.49^{\circ} \mathrm{C}$ to $101.9^{\circ} \mathrm{C}$, and temperature amplitude varies from $6.07{ }^{\circ} \mathrm{C}$ to $56.16^{\circ} \mathrm{C}$. We use the ceiling values of the minimum and maximum mean temperatures. The minimum amplitude is considered as 10. A central composite factorial DoE method is applied to this design space to provide eight design points. Additionally four points are obtained through Latin hypercube based sampling DoE technique. The twelve experimental points include eight central composite factorial points and four Latin hypercube sampling points. The DoE points are listed in Table 4.

Table 4 also shows the dimensionless scaled values of design variables over the range -1 to 1 in the following generation of the reduced order models. The values of accumulated plastic strain are used in Eq. (17) to predict the mean life time to failure of the wire bond structure due to lift off. The ROMs are generated for the characteristic performances for the logarithmic value of accumulated plastic strain.

\subsection{Kriging reduced order model}

Kriging is a method of interpolation, which predicts unknown characteristic value of a particular point based on characteristic data observed at known points. It minimizes the error of predicted characteristic values, which are estimated by distribution of observed data. Kriging reduced order model is promising for building accurate global approximation of design space. The Kriging model is defined as
Table 3 Cycles from rainflow counting algorithm for the temperature profile at the interface

\begin{tabular}{lrrlrrl}
\hline $\begin{array}{l}\text { Cycle } \\
\text { name }\end{array}$ & \multicolumn{1}{l}{ Mean } & Amplitude & Cycles & $\begin{array}{l}\text { Start } \\
\text { time }\end{array}$ & Period & $\begin{array}{l}\text { Peak/ } \\
\text { valley }\end{array}$ \\
\hline$C_{1}$ & 67.4900 & 6.0680 & 1.0 & 5.6 & 9.6 & Valley \\
$C_{2}$ & 100.5720 & 11.9600 & 1.0 & 15.2 & 10.4 & Valley \\
$C_{3}$ & 88.4705 & 6.5985 & 1.0 & 40.8 & 8.8 & Peak \\
$C_{4}$ & 90.0470 & 10.4310 & 1.0 & 55.2 & 10.4 & Valley \\
$C_{5}$ & 94.6130 & 19.0560 & 1.0 & 50.4 & 29.6 & Peak \\
$C_{6}$ & 76.6855 & 6.8535 & 1.0 & 80.8 & 8.8 & Peak \\
$C_{7}$ & 88.7090 & 6.6450 & 1.0 & 100.8 & 8.8 & Peak \\
$C_{8}$ & 86.0410 & 18.2700 & 1.0 & 90.4 & 9.6 & Peak \\
$C_{9}$ & 80.4925 & 8.6855 & 1.0 & 125.2 & 10.4 & Valley \\
$C_{10}$ & 86.9830 & 9.9080 & 1.0 & 135.2 & 10.4 & Valley \\
$C_{11}$ & 86.4955 & 6.4985 & 1.0 & 150.8 & 8.8 & Peak \\
$C_{12}$ & 84.2210 & 56.1640 & 0.5 & 0.2 & 50.0 & Peak \\
$C_{13}$ & 101.9320 & 38.4530 & 0.5 & 25.2 & 190.4 & Valley \\
$C_{14}$ & 82.5675 & 19.0885 & 0.5 & 120.4 & 49.6 & Peak \\
$C_{15}$ & 88.3725 & 13.2835 & 0.5 & 145.2 & 29.6 & Valley \\
\hline
\end{tabular}

$\hat{y}(\boldsymbol{X})=\sum_{j=0}^{m} \beta_{j} P_{j}(\boldsymbol{X})+\sum_{i=1}^{n} \alpha_{i} r_{i}$

where $\boldsymbol{X}$ is the vector of the $m$ design variables. $\boldsymbol{X}=\left(x_{1}\right.$, $\left.x_{2}, \cdots, x_{m}\right)$ and $\beta_{j}(j=0,1, \cdots, m)$ are the coefficients of the polynomials $P_{j}(\boldsymbol{X})(j=0,1, \cdots, m)$. In Eq. (19), $\alpha_{i}(i=1,2$, $\cdots, n)$ is the coefficients of the basis function $r_{i}(i=1,2, \cdots$, $n$ ), where $n$ is the number of the DoE points. The polynomial $P_{j}(\boldsymbol{X})$ is linear (i.e., $P_{j}(\boldsymbol{X})=x_{j}, j=1,2, \cdots, m$, and $\left.P_{0}(X)=1\right)$.

The basis function $r_{i}=\varphi\left(\left\|\boldsymbol{X}-\boldsymbol{X}_{i}\right\|\right)$ is called a variogram and has an argument about the absolute distance between points $\boldsymbol{X}$ and $\boldsymbol{X}_{i}$. There are many variogram models available. In this work, the spherical model is utilized as it is suitable for cases with small number of design variables, typically when $m \leq 3$ [30]. If $h_{i}=\left\|\boldsymbol{X}-\boldsymbol{X}_{i}\right\|$ $(i=1,2, \cdots, n)$, the spherical model is defined as

$\varphi\left(h_{i}\right)=\left\{\begin{array}{lcc}0, & \text { if } & h_{i}=0, \\ C_{1}\left(\frac{1.5 h_{i}}{C_{2}}-0.5 \frac{h_{i}^{3}}{C_{2}^{3}}\right), & \text { if } & 0<h_{i} \leq C_{2}, \\ C_{1}, & \text { if } & h_{i}>C_{2},\end{array}\right.$

where $C_{1}$ and $C_{2}$ are the variogram coefficients. The unknown coefficients in Kriging ROMs, $\beta_{j}(j=0,1, \cdots, m)$, $\alpha_{i}(i=1,2, \cdots, n), C_{1}$ and $C_{2}$ are computed so that the variation errors of the predicted and observed data are minimized [30].

The Kriging ROM, $K_{\varepsilon_{\mathrm{p}}}$ for the accumulated plastic strain on the slice of aluminium wire bond is defined as 
Table 4 DoE points and predicted wire bond model responses from FEA

\begin{tabular}{|c|c|c|c|c|c|c|}
\hline \multirow[t]{2}{*}{ DoE points No. } & \multicolumn{2}{|c|}{ Temperature excursion $(\Delta T)$} & \multicolumn{2}{|c|}{ Mean value of temperature $\left(T_{\mathrm{m}}\right)$} & \multirow{2}{*}{$\begin{array}{l}\text { Accumulated } \\
\text { plastic strain }\left(\Delta \varepsilon_{\mathrm{p}}\right)\end{array}$} & \multirow{2}{*}{$\begin{array}{l}\text { Logarithm of } \\
\text { accumulated } \\
\text { plastic strain }\left(\ln \left(\Delta \varepsilon_{\mathrm{p}}\right)\right)\end{array}$} \\
\hline & Actual & Scaled & Actual & Scaled & & \\
\hline 1 & 10 & -1 & 67 & -1 & $3.847990 \times 10^{-7}$ & -14.770544 \\
\hline 2 & 10 & -1 & 102 & 1 & $4.311272 \times 10^{-7}$ & -14.656863 \\
\hline 3 & 57 & 1 & 67 & -1 & $6.475778 \times 10^{-3}$ & -5.039687 \\
\hline 4 & 57 & 1 & 102 & 1 & $6.534895 \times 10^{-3}$ & -5.0305998 \\
\hline 5 & 10 & -1 & 84.5 & 0 & $4.069855 \times 10^{-7}$ & -14.714488 \\
\hline 6 & 57 & 1 & 84.5 & 0 & $5.224363 \times 10^{-3}$ & -5.254422 \\
\hline 7 & 33.5 & 0 & 67 & -1 & $2.709545 \times 10^{-3}$ & -5.910974 \\
\hline 8 & 33.5 & 0 & 102 & 1 & $3.388047 \times 10^{-3}$ & -5.687502 \\
\hline 9 & 10.244 & -0.9896 & 73.111 & -0.6508 & $4.680694 \times 10^{-7}$ & -14.574649 \\
\hline 10 & 42.411 & 0.3792 & 93.007 & 0.4861 & $4.148474 \times 10^{-3}$ & -5.485015 \\
\hline 11 & 31.472 & -0.0863 & 76.347 & -0.4659 & $2.401401 \times 10^{-3}$ & -6.031703 \\
\hline 12 & 52.166 & 0.7943 & 100.789 & 0.9308 & $5.742838 \times 10^{-3}$ & -5.159802 \\
\hline
\end{tabular}

$$
\begin{aligned}
K_{\varepsilon_{\mathrm{p}}}\left(T_{\text {Delta }}, T_{\text {Mean }}\right)= & -8.689+4.822 T_{\text {Delta }}+0.049 T_{\text {Mean }} \\
& +9.8701 \sum_{i=1}^{13} k_{i}^{\varepsilon_{\mathrm{p}}}\left(\frac{1.5 h_{i}}{1.3869}-\frac{0.5 h_{i}^{3}}{2.6678}\right),
\end{aligned}
$$

$h_{i}=\sqrt{\left(T_{\text {Delta }}-\left(T_{\text {Delta }}\right)_{i}\right)^{2}+\left(T_{\text {Mean }}-\left(T_{\text {Mean }}\right)_{i}\right)^{2}}$.

The advantage of Kriging reduced order model is its ability to predict the variance $\sigma(\cdot)$ at each estimation point. The prediction intervals of the Kriging response surface model can be constructed as

$A=\left(K_{\varepsilon_{\mathrm{p}}}(\cdot)-1.96 \sigma(\cdot), K_{\varepsilon_{\mathrm{p}}}(\cdot)+1.96 \sigma(\cdot)\right)$,

where $A$ is the nominal $95 \%$ prediction interval for Kriging model $K_{\varepsilon_{\mathrm{p}}}(\cdot)$ under the assumption that it is Gaussian and it standard deviation is $\sigma(\cdot)$. A $95 \%$ prediction interval for each estimation point is generated based on the assumption that each Kriging estimation point follows a normal distribution with this square root of variance value (standard deviation) and the Kriging estimation value at that point as mean value. The plot of prediction interval at various points will create a bandwidth. By varying only one design variable at nominal value $(0)$, we can generate a bandwidth plot for each design variable. The prediction intervals of the Kriging ROM for the amplitude of temperature load and mean value of temperature load are plotted in Fig. 6.

\subsection{Radial basis function (RBF) reduced order model}

The RBF interpolation is popular for scattered data interpolating. The primary advantage of $\mathrm{RBF}$ is that the resulting linear system from the RBF interpolation is invertible in most situations. A response $y(x)$ in a domain has a set of data points $\left\{x_{i}\right\}_{i=0,1, \cdots, n}$. The nodal response values are $y_{i=0,1, \cdots, n}$ at data points. Radial basis response surface constructs the reduced order model function to pass through all these training data points using radial basis function $\phi\left(\left\|\boldsymbol{X}-\boldsymbol{X}_{j}\right\|\right)$ and a polynomial $P(\boldsymbol{X})$.

$$
\begin{aligned}
y(\boldsymbol{X}) & =\sum_{i=1}^{n} a_{i} \phi\left(\left\|\boldsymbol{X}-\boldsymbol{X}_{i}\right\|\right)+\sum_{j=1}^{m} b_{j} P_{j}(\boldsymbol{X}) \\
& =B^{\mathrm{T}}(\boldsymbol{X}) a+P^{\mathrm{T}}(\boldsymbol{X}) b
\end{aligned}
$$

where $a_{i}$ is the coefficient of $\phi\left(\left\|\boldsymbol{X}-\boldsymbol{X}_{j}\right\|\right)$ and $b_{j}$ is the coefficient of the polynomial term $P_{j}[31,32]$. A radial basis function has the following general form:

$\phi\left(\left\|\boldsymbol{X}-\boldsymbol{X}_{j}\right\|\right)=\phi\left(r_{i}\right)$,

where $r_{i}$ is the Euclidean distance between interpolation points $x$ and $x_{i}$. Several classes of radial basis function may be chosen for $\varphi$ [31]. In this paper, we use Gaussian radial basis function

$\phi(r)=\mathrm{e}^{-\beta r^{2}}$,

where Gaussian radial basis function is the most commonly used with $\beta=0.01$. Polynomial $P$ is usually a lower order polynomial, and in this paper we uses a linear form. The radial basis reduced order model for the accumulated plastic strain on aluminium wire bond slice attached to the silicon chip $R_{\varepsilon_{\mathrm{p}}}$ is

$$
\begin{aligned}
R_{\varepsilon_{\mathrm{p}}}\left(T_{\text {Delta }}, T_{\text {Mean }}\right)= & -32.0322+93.0576 T_{\text {Delta }} \\
& +6.5957 T_{\text {Mean }}+\sum_{i=1}^{n} r_{i}^{\varepsilon_{\mathrm{p}}} \mathrm{e}^{-0.01 h_{i}^{2}}
\end{aligned}
$$

where $i=1,2, \cdots, 12$, and $h_{i}$ is as in Eq. (21). The values of the coefficients are listed in Table 5. 

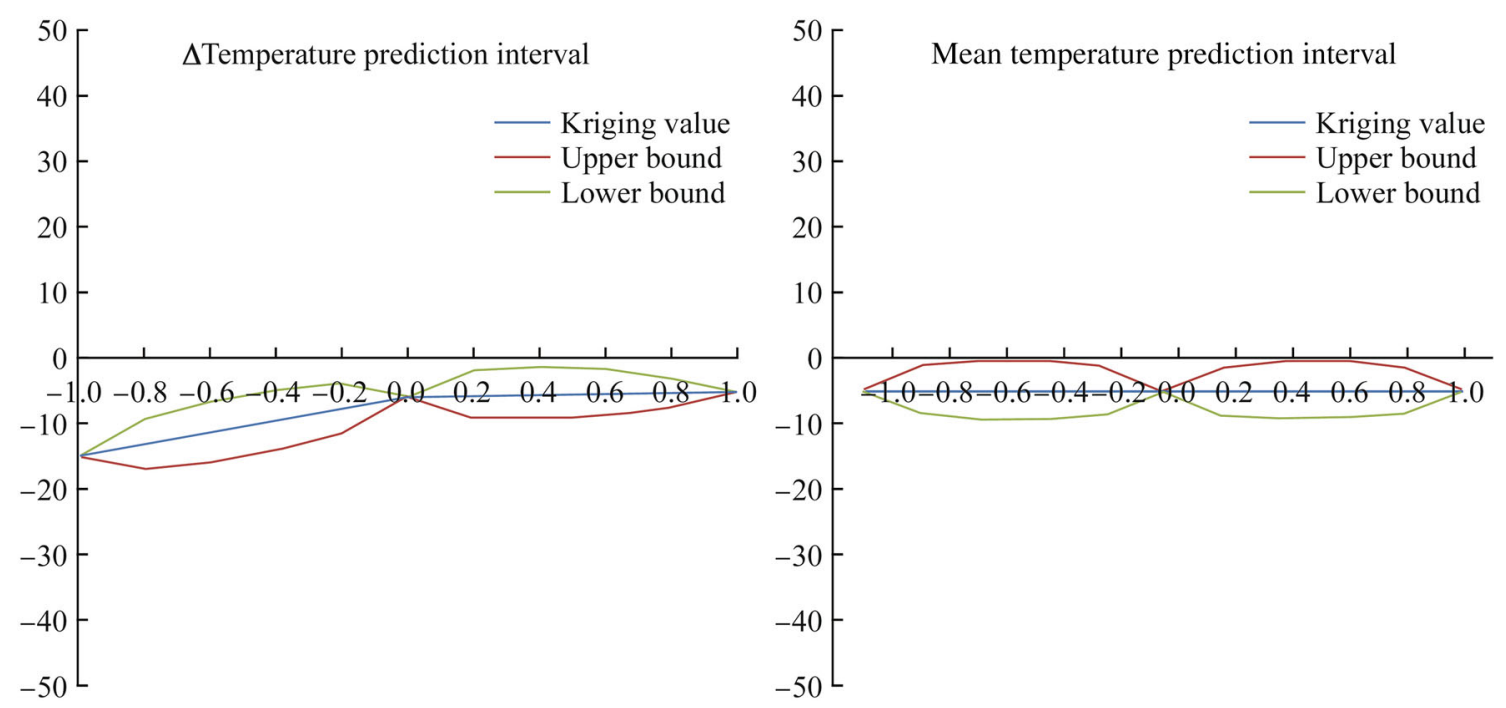

Fig. 6 Upper and lower bounds of the Kriging ROM for the amplitude of the load temperature and mean temperature

Table 5 Coefficients for Kriging and RBF reduced order models

Wire bond model DoE scaled value Coefficients of reduced order of design variable

\begin{tabular}{|c|c|c|c|}
\hline & \multirow[b]{2}{*}{$\begin{array}{l}\text { Kriging } \\
k_{i}{ }_{\mathrm{p}}\end{array}$} & \multirow[b]{2}{*}{$\begin{array}{l}\text { Radial basis } \\
r_{i}^{c}\end{array}$} \\
\hline$\overline{\left(T_{\text {Delta }}\right)_{i}}$ & $\left(T_{\text {Mean }}\right)_{i}$ & & \\
\hline-1 & -1 & 0.119 & $24,966,978.932$ \\
\hline-1 & 1 & 0.143 & $-3,704,831.701$ \\
\hline 1 & -1 & 0.127 & $-140,690.375$ \\
\hline 1 & 1 & 0.166 & $-23,374,973.999$ \\
\hline-1 & 0 & 0.098 & $23,037,927.678$ \\
\hline 1 & 0 & 0.137 & $4,181,062.763$ \\
\hline 0 & -1 & -0.206 & $-11,754,136.474$ \\
\hline 0 & 1 & -0.302 & $854,952.017$ \\
\hline-0.9896 & -0.6508 & 0.055 & $-47,099,621.802$ \\
\hline 0.3792 & 0.4861 & -0.076 & $-35,050,173.622$ \\
\hline-0.0863 & -0.4659 & -0.250 & $26,984,797.475$ \\
\hline 0.7943 & 0.9308 & -0.01 & $41,098,709.108$ \\
\hline
\end{tabular}

\section{Life time consumed}

By applying the rainflow cycle counting algorithm to the random temperature profile of the wirebond and aluminium pad junction, the individual temperature cycles for various amplitudes and their quantities are extracted. The reduced order model corresponds to the accumulated inelastic strain as a function of amplitude, and mean value of temperature cycle is derived. The summation of these reciprocals of the fatigue life is the accumulated fatigue damage by LDR, as shown in Fig. 6. Similar methodology is applied for other fatigue damage models (see Fig. 7).

It is assumed that the random load current is applied to the wire, as shown in Fig. 4. This load profile is applied for five times, then the accumulated damage $(D)$ of the aluminium wire and aluminium plate interface is predicted using the four cumulative fatigue damage methods (Eqs. 1-14). The results are summarized in Tables 6 and 7. The contribution from small temperature amplitude cycles $\left(\Delta T<10^{\circ} \mathrm{C}\right)$ is ignored. Generally, these amplitude temperature cycles often generate very small accumulated strain per cycle, and the resulting contributions are insignificant to the whole life time consumption value.

Apart from DLDR, the other three models predict more or less the same wear out ratio for both types of ROM models. The reference fatigue lives $\left(N_{\text {Ref }}\right)$ for both DCA and DDCA type fatigue models are set as the lowest fatigue life in the block. For DLDR the lowest fatigue life $\left(N_{\text {Low }}\right)$ and the highest fatigue life $\left(N_{\text {High }}\right)$ are based on the model ROM. We use these values due to the unavailable fatigue life data of aluminium for DLDR in the literatures. To summarize the results, the LDR, DCA, and DDCA predict almost the similar results. DLDR predicts slightly higher wear out ratio. The ROM models (Kriging and radial basis) are interpolation types. Within the two physics of failure based reduced models, Kriging ROM tends to produce slightly larger accumulated damage $\left(D_{\text {Total }}\right)$ in comparison with radial basis ROM. Kriging ROM's total accumulated damage of $0.0027 \%$ is less in comparison with total accumulated damage of $0.0032 \%$ by radial basis ROM. The percentage differences between the total accumulated damages of LDR and DCA by Kriging and radial basis ROMs are $8 \%$ and $5 \%$ respectively. Likewise the percentage differences of total accumulated damage percentages between LDR and DDCA by Kriging and radial basis ROMs are both $1 \%$. 


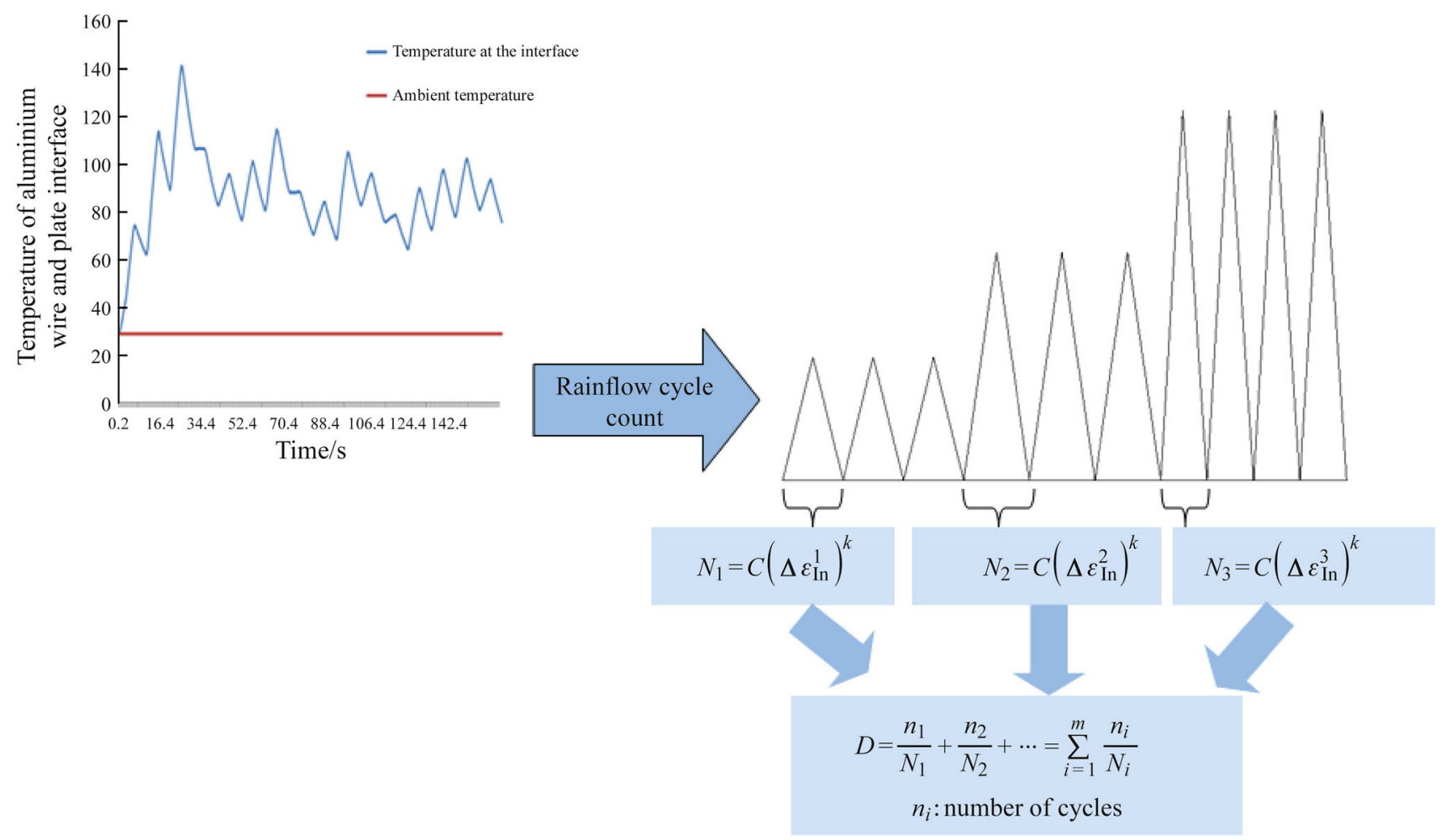

Fig. 7 Accumulated damage by LDR cumulative fatigue damage model

Table 6 Accumulated damage by various cumulative fatigue damage models using the Kriging ROM

\begin{tabular}{|c|c|c|c|c|c|c|c|c|c|c|}
\hline & $\Delta T /{ }^{\circ} \mathrm{C}$ & $T_{\mathrm{m}} /{ }^{\circ} \mathrm{C}$ & $\begin{array}{l}\text { No. } \\
\text { of } \\
\text { cycle }\end{array}$ & $\ln \left(\Delta \varepsilon_{\mathrm{p}}\right)$ & $\Delta \varepsilon_{\mathrm{p}}$ & $N_{\mathrm{f}}$ & $D(\mathrm{LDR})$ & $D$ (DCR) & $D$ (DLDR) & $D$ (DDCA) \\
\hline$C_{2}$ & 11.96 & 100.572 & 5 & -13.993 & $8.375 \times 10^{-7}$ & $3.22 \times 10^{8}$ & $1.553 \times 10^{-8}$ & 0 & $1.573 \times 10^{-8}$ & $2.702 \times 10^{-10}$ \\
\hline$C_{4}$ & 10.431 & 90.074 & 5 & -14.423 & $5.447 \times 10^{-7}$ & $5.88 \times 10^{8}$ & $8.503 \times 10^{-9}$ & 0 & $8.593 \times 10^{-9}$ & $1.267 \times 10^{-10}$ \\
\hline$C_{5}$ & 19.056 & 94.613 & 5 & -11.546 & $9.67 \times 10^{-6}$ & $1.048 \times 10^{7}$ & $4.771 \times 10^{-7}$ & 0 & $5.017 \times 10^{-7}$ & $2.046 \times 10^{-8}$ \\
\hline$C_{8}$ & 18.27 & 86.041 & 5 & -11.932 & $6.578 \times 10^{-6}$ & $1.797 \times 10^{7}$ & $2.782 \times 10^{-7}$ & 0 & $2.895 \times 10^{-7}$ & $1.031 \times 10^{-8}$ \\
\hline$C_{12}$ & 56.164 & 84.221 & 2.5 & -5.327 & $4.86 \times 10^{-3}$ & $1.733 \times 10^{3}$ & $1.443 \times 10^{-3}$ & $1.443 \times 10^{-3}$ & $6.22 \times 10^{-3}$ & $1.442 \times 10^{-3}$ \\
\hline$C_{13}$ & 38.453 & 101.932 & 2.5 & -5.375 & $4.63 \times 10^{-3}$ & $1.854 \times 10^{-3}$ & $1.348 \times 10^{-3}$ & $1.125 \times 10^{-3}$ & $5.601 \times 10^{-3}$ & $1.313 \times 10^{-3}$ \\
\hline$C_{14}$ & 19.089 & 82.568 & 2.5 & -11.495 & $1.018 \times 10^{-5}$ & $9.753 \times 10^{6}$ & $2.563 \times 10^{-7}$ & 0 & $2.698 \times 10^{-7}$ & $1.12 \times 10^{-8}$ \\
\hline$C_{15}$ & 13.284 & 88.373 & 2.5 & -13.636 & $1.197 \times 10^{-6}$ & $1.953 \times 10^{8}$ & $1.28 \times 10^{-8}$ & 0 & $1.3 \times 10^{-8}$ & $2.534 \times 10^{-10}$ \\
\hline$D_{\text {Total }}$ & & & & & & & $2.8 \times 10^{-5}$ & $2.6 \times 10^{-5}$ & $1.18 \times 10^{-4}$ & $2.8 \times 10^{-5}$ \\
\hline
\end{tabular}

\subsection{Prediction uncertainty}

The input design variables have some uncertainty associated with them. The ROM that depends on the design variables will also have uncertainty associated. The input variables $\left(\Delta T, T_{\mathrm{m}}\right)$ are deterministic values, which are extracted from the rainflow counting algorithm. Main advantage of Kriging ROM is of quantifying the estimation variance, which will define the precision of the resulting estimation as in Eq. (22).
The Kriging variances $(\sigma)$ of prediction points and the resulting upper and lower bounds of the Kriging ROM prediction estimation are listed in Table 8 . The bounds are $95 \%$ prediction interval as in Eq. (22). Using the bounds of each prediction we can evaluate the prediction bound of the Kriging lifetime of each fatigue life model, as shown in Table 9.

The ranges of accumulated damage prediction intervals by LDR, DCA, and DLDR are respectively $0.0089 \%$, $0.0801 \%, 0.113 \%$. Hence, the accumulated damage 
Table 7 Accumulated damage by various cumulative fatigue damage models using the radial ROM

\begin{tabular}{|c|c|c|c|c|c|c|c|c|c|c|}
\hline & $\Delta T /{ }^{\circ} \mathrm{C}$ & $T_{\mathrm{m}} /{ }^{\circ} \mathrm{C}$ & $\begin{array}{l}\text { No. } \\
\text { of } \\
\text { cycle }\end{array}$ & $\ln \left(\Delta \varepsilon_{\mathrm{p}}\right)$ & $\Delta \varepsilon_{\mathrm{p}}$ & $N_{\mathrm{f}}$ & $D(\mathrm{LDR})$ & $D(\mathrm{DCR})$ & $D$ (DLDR) & $D$ (DDCA) \\
\hline$C_{2}$ & 11.96 & 100.572 & 5 & -13.357 & $1.582 \times 10^{-6}$ & $1.322 \times 10^{8}$ & $3.782 \times 10^{-8}$ & 0 & $3.855 \times 10^{-8}$ & $7.997 \times 10^{-10}$ \\
\hline$C_{4}$ & 10.431 & 90.074 & 5 & -14.414 & $5.496 \times 10^{-7}$ & $5.806 \times 10^{8}$ & $8.612 \times 10^{-9}$ & 0 & $8.704 \times 10^{-9}$ & $1.243 \times 10^{-10}$ \\
\hline$C_{5}$ & 19.056 & 94.613 & 5 & -9.659 & $6.385 \times 10^{-5}$ & $7.461 \times 10^{5}$ & $6.702 \times 10^{-6}$ & 0 & $7.722 \times 10^{-6}$ & $5.769 \times 10^{-7}$ \\
\hline$C_{8}$ & 18.27 & 86.041 & 5 & -9.951 & $4.768 \times 10^{-5}$ & $1.123 \times 10^{6}$ & $4.453 \times 10^{-6}$ & 0 & $5.025 \times 10^{-6}$ & $3.408 \times 10^{-7}$ \\
\hline$C_{12}$ & 56.164 & 84.221 & 2.5 & -5.255 & $5.221 \times 10^{-3}$ & $1.567 \times 10^{3}$ & $1.595 \times 10^{-3}$ & $1.446 \times 10^{-3}$ & $7.565 \times 10^{-3}$ & $1.579 \times 10^{-3}$ \\
\hline$C_{13}$ & 38.453 & 101.932 & 2.5 & -5.228 & $5.364 \times 10^{-3}$ & $1.509 \times 10^{3}$ & $1.657 \times 10^{-3}$ & $1.657 \times 10^{-3}$ & $8.04 \times 10^{-3}$ & $1.657 \times 10^{-3}$ \\
\hline$C_{14}$ & 19.089 & 82.568 & 2.5 & -9.558 & $7.063 \times 10^{-5}$ & $6.477 \times 10^{5}$ & $3.860 \times 10^{-6}$ & 0 & $4.483 \times 10^{-6}$ & $3.462 \times 10^{-7}$ \\
\hline$C_{15}$ & 13.284 & 88.373 & 2.5 & -12.562 & $3.503 \times 10^{-6}$ & $4.344 \times 10^{7}$ & $5.756 \times 10^{-8}$ & 0 & $5.926 \times 10^{-8}$ & $1.628 \times 10^{-9}$ \\
\hline$D_{\text {Total }}$ & & & & & & & $3.3 \times 10^{-5}$ & $3.1 \times 10^{-5}$ & $1.56 \times 10^{-4}$ & $3.2 \times 10^{-5}$ \\
\hline
\end{tabular}

Table 8 Upper and lower bounds of the the Kriging ROM prediction

\begin{tabular}{lllll}
\hline & $\begin{array}{l}\text { Kriging } \\
\text { prediction }(\ln \\
\left.\left(\Delta \varepsilon_{\mathrm{p}}\right)\right)\end{array}$ & $\begin{array}{l}\text { Variance } \\
\left(\sigma^{2}\right)\end{array}$ & $\begin{array}{l}\text { Lower bound } \\
(\ln \\
\left.\left(\Delta \varepsilon_{\mathrm{p}}\right)+1.96 \sigma\right)\end{array}$ & $\begin{array}{l}\text { Upper bound } \\
\left(\ln \left(\Delta \varepsilon_{\mathrm{p}}\right)-\right. \\
1.96 \sigma)\end{array}$ \\
\hline$C_{2}$ & -13.993 & 0.426 & -12.714 & -15.272 \\
$C_{4}$ & -14.422 & 0.492 & -13.048 & -15.798 \\
$C_{5}$ & -11.546 & 0.716 & -9.888 & -13.204 \\
$C_{8}$ & -11.932 & 0.592 & -10.424 & -13.440 \\
$C_{12}$ & -5.327 & 0.083 & -4.762 & -5.892 \\
$C_{13}$ & -5.375 & 0.335 & -4.241 & -6.509 \\
$C_{14}$ & -11.495 & 0.558 & -10.031 & -12.959 \\
$C_{15}$ & -13.636 & 0.465 & -12.299 & -14.973 \\
\hline
\end{tabular}

Table 9 Kriging lifetime prediction upper and lower bounds

\begin{tabular}{lrll}
\hline$D_{\text {Total }}$ & \multicolumn{1}{l}{$\begin{array}{l}\text { Lower } \\
\text { bound }\end{array}$} & $\begin{array}{l}\text { Kriging ROM accumulated } \\
\text { damage }\left(D_{\text {Total }}\right)\end{array}$ & Upper bound \\
\hline LDR & $9 \times 10^{-6}$ & $2.8 \times 10^{-5}$ & $9.8 \times 10^{-5}$ \\
DCA & $4 \times 10^{-7}$ & $2.6 \times 10^{-5}$ & $8.02 \times 10^{-4}$ \\
DLDR & $2.5 \times 10^{-5}$ & $1.18 \times 10^{-4}$ & $1.155 \times 10^{-3}$ \\
DDCA & $5 \times 10^{-6}$ & $2.8 \times 10^{-5}$ & - \\
\hline
\end{tabular}

prediction $95 \%$ confidence interval by LDR is lower in comparison with the confidence intervals of other methods.

\section{Conclusions}

This paper has discussed the mean fatigue lifetime prediction of wirebond structure model under the variable amplitude loading conditions. The predictions by various nonlinear cumulative fatigue models are discussed. Variable amplitude loading currents are applied to a simplified wirebond structure finite element model. Physics of failure based approach consists of high fidelity modelling or numerical simulation, fast design evaluation models or reduced order models, and DoE methods are utilized. High fidelity modelling consists of electro-thermo-mechanical analysis in FEM setup. Physics of failure based reduced order models that can capture the nonlinear behaviour of the blackbox function using Kriging and radial basis are also utilized. The rainflow counting algorithm is utilized to count the temperature profile of the wirebond structure and the temperature cycles. The mean accumulated damage prediction of the wire bond structure over this usage period is predicted by DDCA, DCA, DLDR and LDR. The LDR, DCA and DDCA predict almost the similar wear out percentage values for the specific random load profile. The prediction error bands are also evaluated for four models in the case of Kriging ROM. The LDR accumulated damage prediction $95 \%$ confidence interval is lower than other methods.

The parameters selected for cumulative models are based on the publically available data in the literatures, which will hinder the accuracy of the results to a certain extent. Ideally, these parameter data are needed to be obtained through the experimental results for the particular material, which will increase the accuracy.

\section{References}

1. Held M, Jacob P, Nicoletti G, Scacco P, Poech MH (1999) Fast power cycling test for insulated gate bipolar transistor modules in traction application'. Int J Electron 86(10):1193-1204

2. Hua L, Bailey C, Yin C (2009) Design for reliability of power electronics modules. Microelectron Reliab 49:1250-1255

3. Ramminger S, Türkes P, Wachutka G (1998) Crack mechanism in wire bonding joints'. Microelectron Reliab 38:1301-1305

4. Ramminger S, Seliger N, Wachutka G (2000) Reliability model for $\mathrm{Al}$ wire bonds subjected to heel crack failures. Microelectron Reliab 40:1521-1525

5. Bielen J, Gommans JJ, Theunis F (2006) Prediction of high cycle fatigue in aluminum bond wires: a physics of failure approach 
combining experiments and multi-physics simulations. In: The 7th international conference on thermal, mechanical and multiphysics simulation and experiments in micro-electronics and micro-systems, 2006. EuroSime 2006. IEEE, New York, pp 1-7

6. Celnikier Y, Benabou L, Coquery G (2011) Investigation of heel crack mechanism in Al connections for power electronics modules. Microelectron Reliab 51:965-974

7. Hager C (2000) Lifetime estimation of aluminum wire bonds based on computational plasticity. Dissertation, Swiss Federal Institute of Technology, Hartung-Gorre

8. Van Driel WD, Van Silfhout RBR, Zhang GQ (2008) Reliability of wirebonds in micro-electronic packages. Microelectron Int 25(2):15-22

9. Meyyappan KN, Hansen P, McCluskey P (2003) Wire flexure fatigue model for asymmetric bond height. In: Proceedings of IPACK conference, Hawai, 2003

10. Shinohara K, Yu Q (2010) Evaluation of fatigue life of semiconductor power device by power cycle test and thermal cycle test using finite element analysis. Engineering 2:1006-1018

11. Kostandyan EE, Sorensen J (2011) Reliability assessment of solder joints in power electronic modules by crack damage model for wind turbine applications. Energies 4:2236-2248

12. White M (2008) Microelectronics reliability: physics-of-failure based modeling and lifetime evaluation. http://nepp.nasa.gov. Assessed 6 July 2011

13. Yin CY, Lu H, Musallam M et al (2008) A physics-of-failure based prognostic method for power modules. In: Electronics packaging technology conference (EPTC) 2008, Singapore. IEEE, New York, pp 1190-1195

14. Fatemi A, Yang L (1998) Cumulative fatigue damage and life prediction theories: a survey of the state of the art for homogeneous materials. Int J Fatigue 20(1):9-34

15. Miner MA (1945) Cumulative damage in fatigue. J Appl Mech 12(3):159-164

16. Manson SS, Halford GR (1981) Practical implementation of the double linear damage rule and damage curve approach for treating cumulative fatigue damage. Int J Fract 17(2):169-192

17. Manson SS, Freche JC, Ensign CR (1967) Application of a double linear damage rule to cumulative fatigue. Paper from fatigue crack propagation, ASTM STP NO 415:384-412

18. Halford GR (1997) Cumulative fatigue damage modeling: crack nucleation and early growth. Int J Fatigue 19:S253-S260
19. Nikolaidis E, Ghiocel DM, Singhal S (eds) (2007) Engineering design reliability applications: for the aerospace, automotive and ship industries. CRC Press, Boca Raton

20. Lu H, Loh WS, Bailey C et al (2008) Computer modelling analysis of the globtop's effects on aluminium wirebond reliability. In: 2nd Electronics system-integration technology conference (ESTC). IEEE, New York, pp 1369-1374

21. ANSYS T M (2009) Academic research advanced version 12.0. ANSYS T M, Canonsburg

22. Khan WA, Culham JR (2008) Modelling of cylindrical pin-fin heat sinks for electronic packaging. IEEE Trans Compon Packag Technol 31(3):536-545

23. Holman JP (2010) Heat transfer, 10th edn. McGraw-Hill, New York

24. Lee WW, Nguyen LT, Selvaduray GS (2000) Solder joint fatigue models: a review and applicability to chip scale packages. Microelectron Reliab 40:231-244

25. Syed A (2004) Accumulated creep starin and energy density based thermal fatigue life prediction models for $\mathrm{SnAgCu}$ solder joints. In: Proceedings of the 54th electronic components and technology conference, June, 2004, pp 737-746

26. Matsuishi M, Endo T (1968) Fatigue of metals subjected to varying stress. In: Paper presented to Japan Society of Mechanical Engineers, Fukuoka, Japan, pp 37-40

27. Downing SD, Socie DF (1982) Simple rainflow counting algorithms. Int J Fatigue 4:31-40

28. Standard A. E1049 (1985) Standard practices for cycle counting in fatigue analysis, Philadelphia

29. Stoyanov S, Rajaguru P, Bailey C (2010) Reduced order modelling for reliability optimisation of advanced micro-systems. In: 2nd International conference on engineering optimization, Lisbon, Portugal, 6-9 September

30. Cressie N (1991) Statistics for spatial data. Wiley, New York

31. Gumerov N, Duraiswami R (2007) Fast radial basis function interpolation via preconditioned Krylov iteration. SIAM J Sci Comput 29(5):1876-1899

32. Wang JG, Liu GR (2002) A point interpolation meshless method based on radial basis function. Int $\mathrm{J}$ Numer Methods Eng 54: 1623-1648 